\title{
Serum milk fat globule-EGF factor 8 protein as a poten- tial biomarker for metabolic syndrome
}

Han Ah Lee ${ }^{1,2, *}$, Jihwan Lim ${ }^{1, *}$, Hyung Joon Joo', Young-Sun Lee', Young Kul Jung', Ji Hoon Kim', Hyunggin An ${ }^{3}$, Hyung Joon Yim', Yoon Tae Jeen', Jong Eun Yeon', Do-Sun Lim'1, Kwan Soo Byun', and Yeon Seok Seo'

'Departments of Internal Medicine, Korea University College of Medicine, Seoul; 'Department of Internal Medicine, Sanggye Paik Hospital, Inje University College of Medicine, Seoul; ${ }^{3}$ Department of Biostatistics, Korea University College of Medicine, Seoul, Korea

\section{Graphical Abstract}

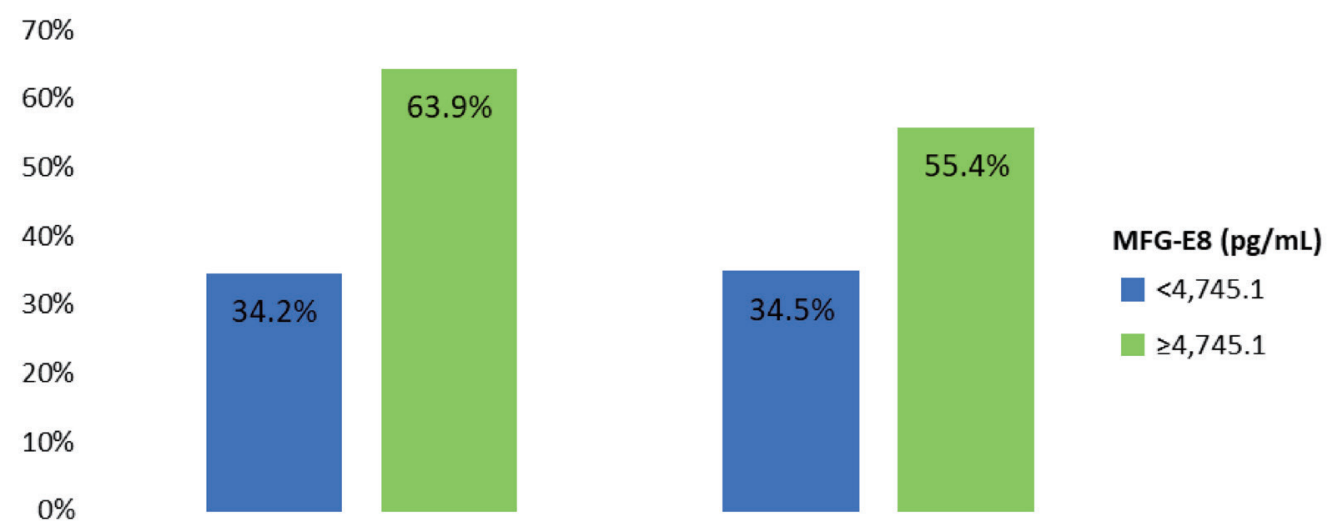

Metabolic syndrome at baseline Development of metabolic syndrome

\section{Abbreviations:}

AUROCs, area under the receiver operating characteristic curves; BMI, body mass index; $\mathrm{BP}$, blood pressure; $\mathrm{Cl}$, confidence interval; $\mathrm{HDL}$, high density lipoprotein; $H R$, hazard ratio; LDL, low density lipoprotein; MFG-E8, milk fat globule-EGF factor-8; OR, odds ratio; S-MAS, Seoul Metabolic Syndrome
Corresponding author: Yeon Seok Seo

Department of Internal Medicine, Korea University College of Medicine, 73 Goryeodae-ro, Seongbuk-gu, Seoul 02841, Korea

Tel: +82-2-920-6608, Fax: +82-2-953-1943

E-mail: drseo@koera.ac.kr

https://orcid.org/0000-0003-4171-6331

\section{Do-Sun Lim}

Department of Internal Medicine, Korea University College of Medicine, 73 Goryeodae-ro, Seongbuk-gu, Seoul 02841, Korea

*Han Ah Lee and Jihwan Lim equally contributed to this work as co-first authors.

Editor: Salvatore Piano, University of Padova Faculty of Medicine and Surgery, Italy
Tel: +82-2-920-6608, Fax: +82-2-927-1478

E-mail: dsImd@kumc.or.kr

https://orcid.org/0000-0001-5751-5177

Copyright $\odot 2021$ by Korean Association for the Study of the Live

This is an Open Access article distributed under the terms of the Creative Commons Attribution Non-Commercial License (http://creativecommons.org/licenses/by-nc/3.0/) which permits unrestricted non-commercial use, distribution, and reproduction in any medium, provided the original work is properly cited. 
Background/Aims: Useful biomarkers for metabolic syndrome have been insufficient. We investigated the performance of serum milk fat globule-EGF factor-8 (MFG-E8), the key mediator of inflammatory pathway, in diagnosis of metabolic syndrome.

Methods: Subjects aged between 30 and 64 years were prospectively enrolled in the Seoul Metabolic Syndrome cohort. Serum MFG-E8 levels were measured at baseline.

Results: A total of 556 subjects were included, comprising 279 women (50.2\%) and 277 men (49.8\%). Metabolic syndrome was diagnosed in 236 subjects $(42.4 \%)$, and the mean MFG-E8 level of subjects with metabolic syndrome was significantly higher than that of subjects without metabolic syndrome $(P<0.001)$. MFG-E8 level was significantly correlated with all metabolic syndrome components and pulse wave velocity (all $P<0.05$ ). Subjects were categorized into two groups according to the best MFG-E8 cut-off value as follows: group 1, MFG-E8 level $<4,745.1 \mathrm{pg} / \mathrm{mL}$ ( $\mathrm{n}=401$, $72.1 \%)$; and group 2, MFG-E8 level $\geq 4,745.1(n=155,27.9 \%)$. At baseline, metabolic syndrome in group 2 was significantly more prevalent than in group 1 ( $63.9 \%$ vs. $34.2 \%, P<0.001)$. During median follow-up of 17 months, metabolic syndrome developed in 122 (38.1\%) subjects among 320 subjects without it at baseline. The incidence of metabolic syndrome in group 2 was significantly higher than that in group 1 ( $55.4 \%$ vs. $34.5 \%, P=0.003)$. On multivariate analysis, MFG-E8 level $\geq 4,745.1 \mathrm{pg} / \mathrm{mL}$ was an independent predictor for diagnosis and development of metabolic syndrome after adjusting other factors (all $P<0.05$ ).

Conclusions: Serum MFG-E8 level is a potent biomarker for the screening and prediction of metabolic syndrome. (Clin Mol Hepatol 2021;27:463-473)

Keywords: Milk fat globule-EGF factor-8; Metabolic syndrome; Biomarkers; Diagnosis

\section{Study Highlights}

Reliable biomarkers for diagnosis and prediction of metabolic syndrome have been required. This prospective cohort study shows that subjects with higher serum MFG-E8 level have a higher risk for metabolic syndrome and further development, which indicates that serum MFG-E8 could be an useful biomarker in screening of metabolic syndrome.

\section{INTRODUCTION}

Metabolic syndrome is a cluster of metabolic derangements caused by central obesity and insulin resistance, and presents increased cardiovascular disease risk as their clinical outcome.' Patients with metabolic syndrome are at increased risk of cardiovascular disease-related mortality and all-cause mortality. Thus, early detection and intervention of metabolic syndrome are imperative. However, frequently, diagnosis of metabolic syndrome is only possible after complications have already begun. ${ }^{2,3}$

Screening metabolic syndrome with a single serum biomarker is difficult, as it encompasses a wide range of disorders that develop at various times. ${ }^{4,5}$ Several diagnostic criteria for metabolic syndrome which designate values for obesity, hypertension, hyperglycemia, triglyceride and high density lipoprotein (HDL)-cholesterol levels, and urine albumin have been outlined. ${ }^{6}$ In addition, novel biomarkers were discovered to aid in earlier diagnosis when clinical signs are not obvious. ${ }^{7-9}$ While numerous studies have evaluat- ed various cytokines and adipokines thought to be useful as biomarkers, no single biomarker alone has been found to be indicative of metabolic syndrome. ${ }^{10}$ Nevertheless, the development of improved biomarkers is needed to identify individuals with metabolic syndrome and decrease the overall prevalence of the disease in general population using an easy and less invasive method.

Although the entire mechanism of metabolic syndrome is yet to be determined, it is suggested that systemic oxidative stress promotes chronic inflammation, resulting in altered endothelial function and impaired lipid metabolism, and affecting insulin sensitivity." Milk fat globule-EGF factor-8 (MFG-E8) is a multifunctional glycoprotein originally identified as part of the milk fat globule membrane which regulates inflammation through integrin-mediated clearance of apoptotic cells. ${ }^{12,13}$ In addition, previous studies reported that MFG-E8 promotes obesity by mediating uptake of dietary fats and serum fatty acids. ${ }^{14,15}$ MFG-E8 also acts as a bridging molecule between phosphatidylserine on extracellular vesicles and integrins on various cells. ${ }^{16,17}$ Extracellular vesicles 
play important roles in intercellular communication by exchanging substances including lipids. ${ }^{18}$ All these pathways that serum MFGE8 involves in are pathogenic processes of metabolic syndrome. Thus, we hypothesized that serum MFG-E8 level could feasibly serve as a biomarker for detecting metabolic syndrome in general population.

Hence, we performed this study on Seoul Metabolic Syndrome (S-MAS) cohort, consisting of subjects aged 30 to 64 years with no history of cardiovascular disease. We investigated the predictive performance of serum MFG-E8 level for the diagnosis and prediction of the development of metabolic syndrome.

\section{MATERIALS AND METHODS}

\section{Subjects}

This is a prospective study on the S-MAS cohort performed by Korea University Anam Hospital in Seoul, Korea. This study originally aimed to investigate significant prognostic factors and develop valuable prognostic models in metabolic syndrome patients. A total of 1,130 subjects between 30 and 64 years of age were enrolled from 25 public healthcare centers from January to September 2014. Subjects with previous history of angina pectoris, myocardial infarction, stroke, or any revascularization were excluded. Medical examination, standardized questionnaire assessment, laboratory and hemodynamic measurements were performed at baseline. A total of 756 participants were visited for follow-up evaluation until November 2017. The exclusion criteria were as follows: 1) insufficient clinical or laboratory data, 2) lost to follow-up, 3) history of any malignancy, 4) history of autoimmune disease, 5) history of organ transplantation, and 6) history of immunosuppressant drugs (Fig. 1). This study protocol followed the ethical guidelines of the 1975 Declaration of Helsinki, and the Institutional Review Board of Korea University Anam Hospital approved this study (IRB No. ED13087). Written informed consent was obtained from each subject.

\section{Definition of metabolic syndrome}

Metabolic syndrome was defined according to the criteria of the joint interim statement of the International Diabetes Federation Task Force on Epidemiology and Prevention, the National Heart, Lung, and Blood Institute, the American Heart Association, the World Heart Federation, the International Atherosclerosis Society, and the International Association for the Study of Obesity in 2009. ${ }^{6}$ Accordingly, metabolic syndrome was defined as involving three of the following five criteria: 1) increased waist circumference ( $\geq 90 \mathrm{~cm}$ in men and $\geq 80 \mathrm{~cm}$ in women), 2) elevated serum triglyceride level ( $\geq 150 \mathrm{mg} / \mathrm{dL}$ ) or drug treatment for elevated triglyceride level, 3) reduced serum HDL-cholesterol level $(<40$ $\mathrm{mg} / \mathrm{dL}$ in men and $<50 \mathrm{mg} / \mathrm{dL}$ in women) or drug treatment for reduced $\mathrm{HDL}$-cholesterol level, 4) elevated blood pressure (BP) $(\geq 130 / 85 \mathrm{mmHg}$ ) or use of antihypertensive medication, and 5) elevated fasting glucose level (fasting plasma glucose $\geq 100$ $\mathrm{mg} / \mathrm{dL}$ ) or drug treatment for elevated glucose level.

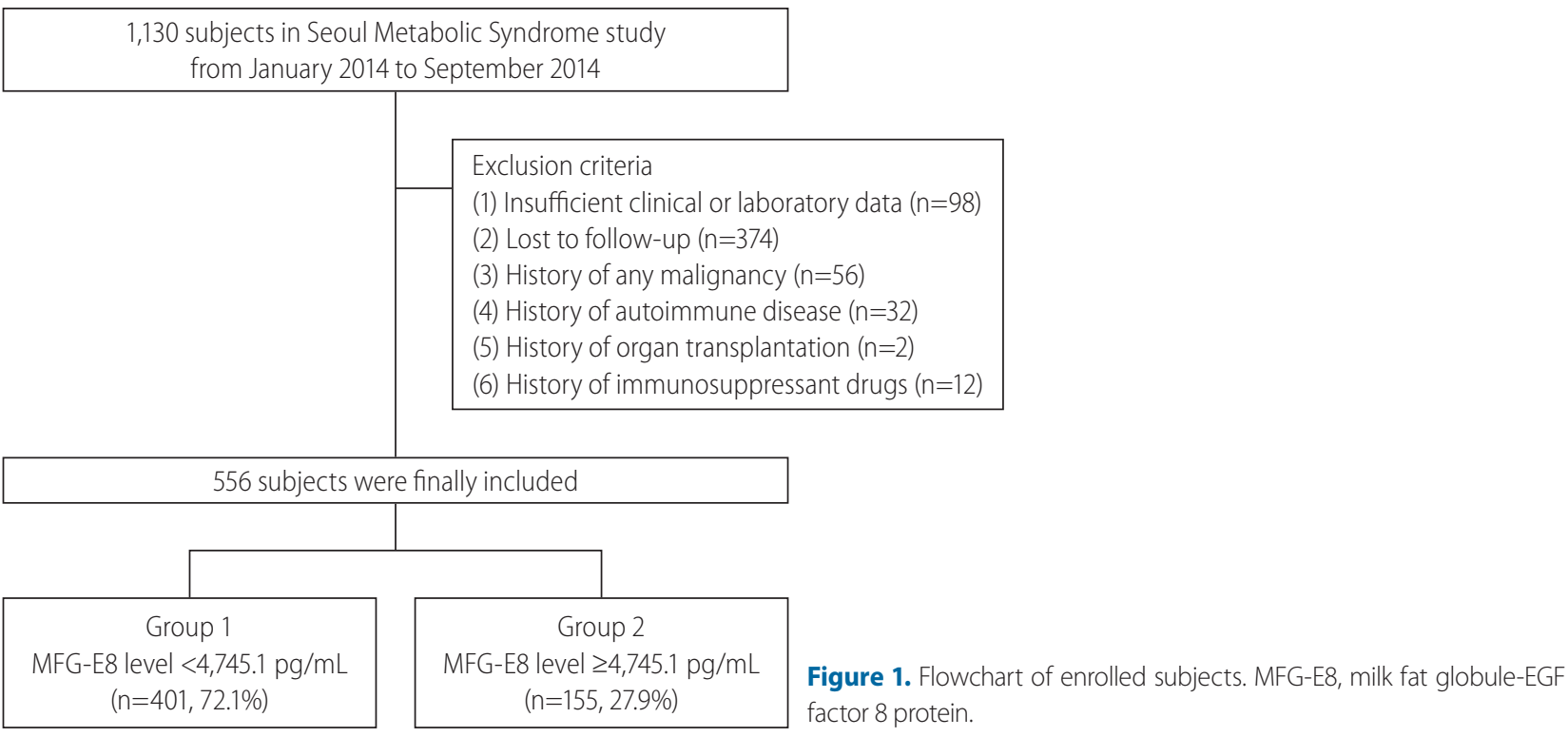




\section{Clinical evaluation and laboratory measurements}

Each subject answered a self-administered questionnaire regarding medical history, anti-diabetic, anti-hyperlipidemic, and anti-hypertensive treatment, alcohol intake, and smoking habit at baseline. Alcohol intake and smoking habit were classified as either current or not current.

Blood samples were collected from each subject after an overnight fast of at least 10 hours at baseline and follow-up examinations. Sitting BP was measured in each arm, and the average value was calculated for analyses. Height and body weight were measured in light clothing without shoes. Pulse wave velocity was measured from carotid-radial artery with non-invasive applanation tonometry. Serum specimens collected at baseline were stored at $-80^{\circ} \mathrm{C}$ until the measurement of serum MFG-E8 levels. Serum MFG-E8 levels was determined using an enzyme-linked immunosorbent assay kit (DFGE80; R\&D Systems, Minneapolis, MN, USA) according to the manufacturer's instructions.

\section{Statistical analysis}

Data are presented as mean (interquartile range) or numbers with percentages. The statistical significance of differences between continuous and categorical variables was compared using

Table 1. Baseline characteristics of the study subjects according to the presence of metabolic syndrome

\begin{tabular}{|c|c|c|c|c|}
\hline Variable & $\begin{array}{l}\text { All subjects } \\
\quad(n=556)\end{array}$ & $\begin{array}{l}\text { Subjects without metabolic } \\
\text { syndrome }(n=320)\end{array}$ & $\begin{array}{l}\text { Subjects with metabolic } \\
\text { syndrome }(n=236)\end{array}$ & $P$-value \\
\hline \multicolumn{5}{|l|}{ Demographic variables } \\
\hline Age (years) & $55.1 \pm 6.5$ & $55.6 \pm 6.1$ & $54.3 \pm 7.0$ & 0.026 \\
\hline Male & $277(49.8)$ & $133(41.6)$ & $144(61.0)$ & $<0.001$ \\
\hline Waist circumference (cm) & $87.9 \pm 8.0$ & $85.0 \pm 7.7$ & $91.8 \pm 6.6$ & $<0.001$ \\
\hline Body mass index $\left(\mathrm{kg} / \mathrm{m}^{2}\right)$ & $25.8 \pm 2.9$ & $25.0 \pm 2.9$ & $26.8 \pm 2.6$ & $<0.001$ \\
\hline Alcohol drinking & $325(58.5)$ & $181(56.6)$ & $144(61.0)$ & 0.292 \\
\hline Current smoker & $212(38.1)$ & $117(36.6)$ & $95(40.3)$ & 0.376 \\
\hline Monthly income (won) & & & & 0.060 \\
\hline None & 109 (19.6) & $72(22.5)$ & $37(15.7)$ & \\
\hline 0 to $2,000,000$ & $105(18.9)$ & $56(17.5)$ & $49(20.8)$ & \\
\hline $2,000,000$ to $4,000,000$ & $290(52.2)$ & $157(49.1)$ & $133(56.4)$ & \\
\hline$>4,000,000$ & $52(9.4)$ & $35(10.9)$ & $17(7.2)$ & \\
\hline Regular exercise & $226(40.6)$ & $141(44.1)$ & $85(36.0)$ & 0.056 \\
\hline Systolic blood pressure (mmHg) & $127.4 \pm 14.1$ & $123.8 \pm 13.5$ & $132.3 \pm 13.4$ & $<0.001$ \\
\hline Diastolic blood pressure $(\mathrm{mmHg})$ & $81.6 \pm 9.3$ & $79.4 \pm 9.0$ & $84.7 \pm 8.7$ & $<0.001$ \\
\hline \multicolumn{5}{|l|}{ Laboratory variables } \\
\hline Total cholesterol (mg/dL) & $198.2 \pm 34.3$ & $198.5 \pm 34.9$ & $197.8 \pm 33.5$ & 0.810 \\
\hline LDL-cholesterol (mg/dL) & $128.2 \pm 33.3$ & $131.0 \pm 32.9$ & $129.5 \pm 33.4$ & 0.124 \\
\hline HDL-cholesterol (mg/dL) & $51.5 \pm 12.5$ & $55.3 \pm 11.9$ & $46.3 \pm 11.4$ & $<0.001$ \\
\hline Triglyceride (mg/dL) & $157.4 \pm 92.7$ & $120.4 \pm 51.3$ & $207.5 \pm 111.0$ & $<0.001$ \\
\hline Glucose (mg/dL) & $100.8 \pm 18.9$ & $96.7 \pm 14.1$ & $106.3 \pm 22.8$ & $<0.001$ \\
\hline Creatinine (mg/dL) & $0.83 \pm 0.16$ & $0.82 \pm 0.15$ & $0.85 \pm 0.16$ & 0.001 \\
\hline High sensitivity CRP (mg/dL) & $1.27 \pm 2.43$ & $1.10 \pm 1.85$ & $1.49 \pm 3.04$ & 0.064 \\
\hline Apolipoprotein Al (mg/dL) & $77.3 \pm 15.8$ & $78.4 \pm 15.3$ & $75.8 \pm 16.5$ & 0.081 \\
\hline MFG-E8 (pg/mL) & $4,195.0 \pm 1,735.7$ & $3,786.0 \pm 1,329.5$ & $4,749.4 \pm 2,044.4$ & $<0.001$ \\
\hline Pulse wave velocity (m/s) & $13.9 \pm 2.1$ & $13.7 \pm 2.2$ & $14.2 \pm 198.5$ & 0.005 \\
\hline
\end{tabular}

Values are presented as mean \pm standard deviation or number $(\%)$.

LDL, low-density lipoprotein; HDL, high-density lipoprotein; CRP, C-reactive protein; MFG-E8, milk fat globule-EGF factor 8 protein. 
Han Ah Lee, et al. MFG-E8 is useful biomarker in metabolic syndrome
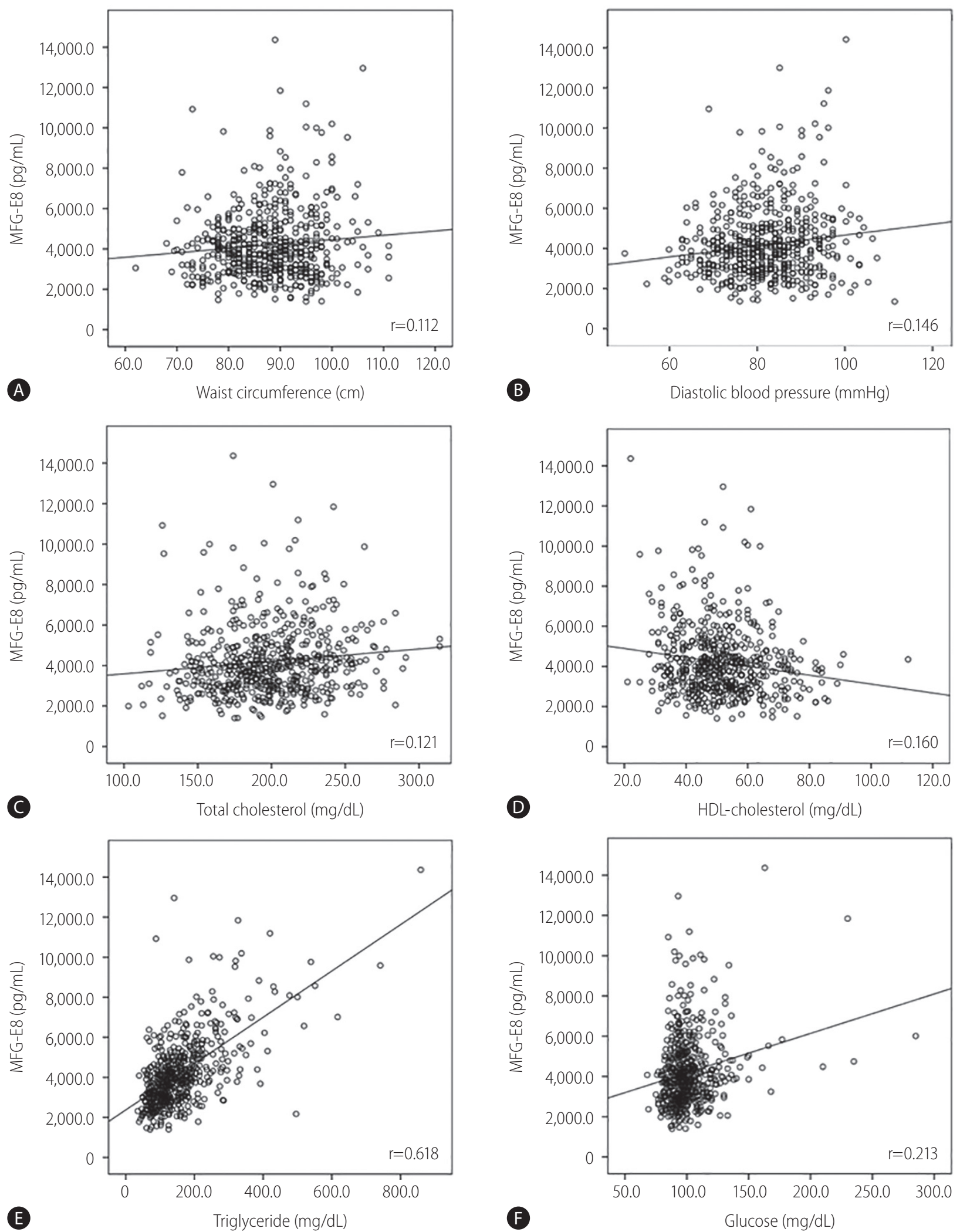

Figure 2. Correlation of serum MFG-E8 level and components of metabolic syndrome. (A) Waist circumference. (B) Diastolic blood pressure. (C) Total cholesterol. (D) HDL-cholesterol. (E) Triglyceride. (F) Glucose levels. MFG-E8, milk fat globule-EGF factor 8 protein; HDL, high-density lipoprotein. 
Student's $t$-test or Mann-Whitney $U$ test and chi-square test, respectively. The predictive accuracy for metabolic syndrome was assessed using area under the receiver operating characteristic curves (AUROCs) as well as 95\% confidence intervals (Cls). Optimal cut-off values were selected to maximize the sum of sensitivity and specificity. The logistic regression analysis was used to evaluate the association between risk factors and metabolic syndrome, and Cox regression analysis was performed for further metabolic syndrome development. Associations were presented as odds ratios (ORs) or hazard ratios (HRs) with $95 \%$ Cls. Statistical analyses were conducted using IBM SPSS Statistics software (version 23.0.0.0; IBM Corporation, Armonk, NY, USA). Two-sided $P$-values $<0.05$ were considered significant.

\section{RESULTS}

\section{Baseline characteristics}

The baseline characteristics of the study subjects are presented in Table 1. A total of 556 subjects were included. The study subjects consisted of 279 women (50.2\%) and 277 men (49.8\%). The mean age of the subjects was 55.1 years. One hundred and three (18.5\%) of the study population had diabetes and 299 (53.8\%) had hypertension, respectively. Among all included subjects, 236 (42.4\%) were defined as having metabolic syndrome at baseline. Subjects with metabolic syndrome had significantly younger age, higher mean body mass index (BMI), higher mean serum creatinine, and higher mean pulse wave velocity than those without metabolic syndrome (all $P<0.05$ ). The proportion of male was higher in subjects with metabolic syndrome than in those without $(P<0.001)$. The mean total cholesterol and low-density lipoprotein (LDL)-cholesterol levels were comparable between two groups (all $P>0.05$ ). The mean serum MFG-E8 level of subjects with metabolic syndrome was significantly higher than that of subjects without metabolic syndrome (4,749.4 vs. 3,786.0 pg/mL, $P<0.001)$.

\section{Correlation of serum MFG-E8 level and metabolic syndrome components}

Serum MFG-E8 level was significantly correlated with waist circumference (Pearson's correlation coefficient, 0.112; $P=0.008$ ), diastolic BP $(0.146, P=0.001)$, serum total cholesterol $(0.121$, $P=0.005)$, HDL-cholesterol $(-0.160, P<0.001)$, triglyceride $(0.618$, $P<0.001)$, glucose $(0.213, P<0.001)$ levels, and pulse wave veloci- ty $(0.134, P=0.002)$ (Fig. 2). In addition, serum MFG-E8 level was significantly higher in subjects with diabetes than in those without $(4,558.5$ vs. $4,112.3 \mathrm{pg} / \mathrm{mL}, P=0.018)$.

\section{Prevalence of metabolic syndrome and patient characteristics at baseline according to the groups classified by serum MFG-E8 level}

The AUROC of the serum MFG-E8 level for the presence of metabolic syndrome at baseline was $0.644(95 \% \mathrm{Cl}, 0.597-0.691$; $P<0.001$ ) (Fig. 3). The optimal cut-off value of the serum MFG-E8 level for the diagnosis of metabolic syndrome was $4,745.1 \mathrm{pg} / \mathrm{mL}$ (sensitivity $41.9 \%$, specificity $82.5 \%$, positive predictive value $63.9 \%$, negative predictive value $65.8 \%$ ). Subjects were categorized into two groups according to the serum MFG-E8 level as follows: group 1, serum MFG-E8 level $<4,745.1 \mathrm{pg} / \mathrm{mL}(\mathrm{n}=401$, 72.1\%); group 2, serum MFG-E8 level $\geq 4,745.1$ ( $n=155,27.9 \%)$.

At baseline, the prevalence of metabolic syndrome in group 2 was significantly higher than that in group $1(63.9 \%$ vs. $34.2 \%$, $P<0.001)$. The prevalence of diabetes, hypertension, and dyslipidemia at baseline was comparable between two groups (all $P>0.05$ ). Male sex was significantly more prevalent in group 2 than in group 1 ( $63.2 \%$ vs. $44.6 \%, P<0.001)$. The mean waist circumference, diastolic BP, total cholesterol, triglyceride, glucose, creatinine levels, and pulse wave velocity of group 2 were signifi-

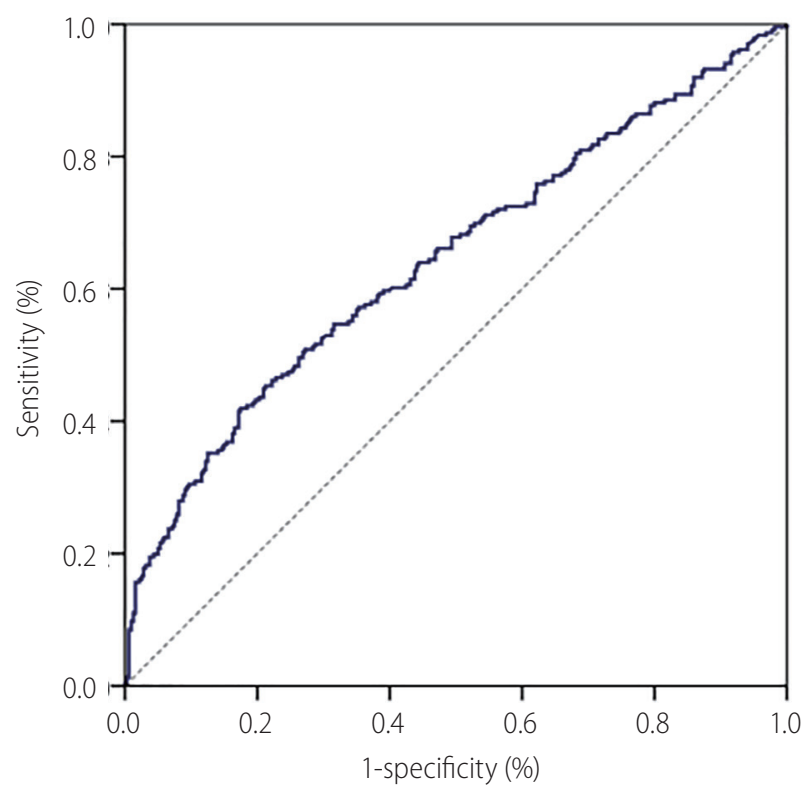

Figure 3. AUROC of MFG-E8 level in the diagnosis of metabolic syndrome at baseline. AUROC, area under the receiver operating characteristic curve; MFG-E8, milk fat globule-EGF factor 8 protein. 
cantly higher than those of group 1 (all $P<0.05$ ). The mean $\mathrm{HDL}$ cholesterol level of group 2 was significantly lower than that of group $1(P<0.001)$ (Table 2$)$.

\section{Prevalence of metabolic syndrome and patient characteristics at follow-up according to the groups classified by serum MFG-E8 level}

During the follow-up period of median 17 months, metabolic syndrome newly developed in 122 (38.1\%) of 320 subjects without metabolic syndrome at baseline. The incidence of metabolic syndrome in group 2 was significantly higher than that in group 1 (55.4\% vs. $34.5 \%, P=0.003$ ). The prevalence of diabetes, hypertension, and dyslipidemia at follow-up was comparable between two groups (all $P>0.05$ ). At follow-up, the mean diastolic BP, total cholesterol, triglyceride levels, and pulse wave velocity of group 2 were significantly higher than those of group 1 (all $P<0.05$ ). The mean HDL-cholesterol level of group 2 was significantly lower than that of group $1(P<0.001)$ (Supplementary Table 1).

The proportions and characteristics of patients according to the changes of the presence of metabolic syndrome are presented in
Supplementary Table 2. The mean MFG-E8 level was 3,636.4 pg/mL in subjects without metabolic syndrome ( $n=198,35.6 \%), 4,029.0$ $\mathrm{pg} / \mathrm{mL}$ in subjects who developed metabolic syndrome at followup ( $n=122,21.9 \%), 4,440.6 \mathrm{pg} / \mathrm{mL}$ in subjects with disappeared metabolic syndrome at follow-up ( $n=72,12.9 \%)$, and 4,885.0 pg/mL in subjects with sustained metabolic syndrome). The mean MFGE8 level of subjects with sustained metabolic syndrome was significantly higher than that of subjects without metabolic syndrome and those who developed metabolic syndrome at followup (all $P<0.001)$.

\section{Predictors for diagnosis and further development of metabolic syndrome}

Independent predictors for diagnosis and further development of metabolic syndrome were investigated (Table 3). In univariate analysis, younger age, male sex, higher BMI, lower LDL-cholester$\mathrm{ol}$, higher creatinine level and MFG-E8 level $\geq 4,745.1 \mathrm{pg} / \mathrm{mL}$ were significantly related to the higher probability for metabolic syndrome at baseline (all $P<0.05$ ). Multivariate analysis showed that male sex $(\mathrm{OR}, 1.818 ; 95 \% \mathrm{Cl}, 1.076-3.074 ; P=0.026)$, higher $\mathrm{BMI}$

Table 2. Baseline characteristics of the study subjects according to the groups classified by serum MFG-E8 level

\begin{tabular}{|c|c|c|c|}
\hline Variable & Group $1(n=401,72.1 \%)$ & Group $2(n=155,27.9 \%)$ & $P$-value \\
\hline Age (years) & $56.0 \pm 6.6$ & $55.1 \pm 6.4$ & 0.977 \\
\hline Male & $179(44.6)$ & $98(63.2)$ & $<0.001$ \\
\hline Waist circumference (cm) & $87.3 \pm 8.0$ & $89.3 \pm 7.7$ & 0.008 \\
\hline Body mass index $\left(\mathrm{kg} / \mathrm{m}^{2}\right)$ & $25.7 \pm 2.9$ & $25.9 \pm 2.9$ & 0.479 \\
\hline Alcohol drinking & $226(56.4)$ & $99(30.5)$ & 0.107 \\
\hline Current smoker & $154(38.4)$ & $58(37.4)$ & 0.830 \\
\hline Systolic blood pressure $(\mathrm{mmHg})$ & $126.8 \pm 14.6$ & $129.1 \pm 12.5$ & 0.087 \\
\hline Diastolic blood pressure (mmHg) & $80.8 \pm 9.4$ & $83.8 \pm 8.5$ & $<0.001$ \\
\hline Total cholesterol (mg/dL) & $195.5 \pm 33.0$ & $205.3 \pm 36.4$ & 0.002 \\
\hline LDL-cholesterol (mg/dL) & $128.2 \pm 31.6$ & $128.3 \pm 37.4$ & 0.988 \\
\hline HDL-cholesterol (mg/dL) & $52.9 \pm 12.9$ & $47.8 \pm 10.7$ & $<0.001$ \\
\hline Triglyceride (mg/dL) & $130.4 \pm 59.5$ & $227.1 \pm 122.3$ & $<0.001$ \\
\hline Glucose (mg/dL) & $99.0 \pm 14.7$ & $105.5 \pm 26.3$ & 0.004 \\
\hline Creatinine (mg/dL) & $0.82 \pm 0.16$ & $0.85 \pm 0.16$ & 0.029 \\
\hline High sensitivity CRP (mg/dL) & $1.21 \pm 2.30$ & $1.40 \pm 2.75$ & 0.415 \\
\hline Apolipoprotein AI (mg/dL) & $77.3 \pm 16.0$ & $77.4 \pm 15.3$ & 0.905 \\
\hline Pulse wave velocity (m/s) & $13.8 \pm 2.1$ & $14.2 \pm 2.2$ & 0.017 \\
\hline
\end{tabular}

Values are presented as mean \pm standard deviation or number (\%).

Group 1, serum MFG-E8 level $<4,745.1 \mathrm{pg} / \mathrm{mL}$; and group 2, serum MFG-E8 level $\geq 4,745.1 \mathrm{pg} / \mathrm{mL}$.

MFG-E8, milk fat globule-EGF factor 8 protein; LDL, low-density lipoprotein; HDL, high-density lipoprotein; CRP, C-reactive protein. 
CLINCAL and MOLECULAR

Volume_27 Number_3 July 2021

Table 3. Predictors for diagnosis and further development of metabolic syndrome

\begin{tabular}{|c|c|c|c|c|c|c|}
\hline \multirow{3}{*}{ Variable } & \multicolumn{3}{|c|}{ Metabolic syndrome at baseline } & \multicolumn{3}{|c|}{ Metabolic syndrome development at follow-up } \\
\hline & \multirow{2}{*}{$\begin{array}{c}\text { Univariate } \\
P \text {-value }\end{array}$} & \multicolumn{2}{|c|}{ Multivariate } & \multirow{2}{*}{$\begin{array}{c}\text { Univariate } \\
P \text {-value }\end{array}$} & \multicolumn{2}{|c|}{ Multivariate } \\
\hline & & OR $(95 \% \mathrm{Cl})$ & $P$-value & & HR (95\% Cl) & $P$-value \\
\hline Age (years) & 0.024 & $0.993(0.964-1.022)$ & 0.628 & 0.016 & $0.981(0.954-1.008)$ & 0.162 \\
\hline Male & $<0.001$ & $1.818(1.076-3.074)$ & 0.026 & 0.051 & & \\
\hline Body mass index $\left(\mathrm{kg} / \mathrm{m}^{2}\right)$ & $<0.001$ & $1.273(1.183-1.369)$ & $<0.001$ & $<0.001$ & $1.137(1.067-1.212)$ & $<0.001$ \\
\hline Alcohol drinking & 0.872 & & & 0.998 & & \\
\hline Current smoker & 0.801 & & & 0.360 & & \\
\hline Regular exercise & 0.057 & & & 0.022 & $0.714(0.502-1.016)$ & 0.061 \\
\hline Total cholesterol (mg/dL) & 0.809 & & & 0.521 & & \\
\hline LDL-cholesterol (mg/dL) & 0.024 & $0.994(0.989-1.000)$ & 0.085 & 0.975 & & \\
\hline Creatinine (mg/dL) & 0.001 & $0.667(0.126-3.525)$ & 0.633 & 0.120 & & \\
\hline High sensitivity CRP (mg/dL) & 0.081 & & & 0.330 & & \\
\hline Apolipoprotein Al (mg/dL) & 0.061 & & & 0.330 & & \\
\hline MFG-E8 $\geq 4,745.1 \mathrm{pg} / \mathrm{mL}$ & $<0.001$ & $3.493(2.298-5.311)$ & $<0.001$ & $<0.001$ & $1.971(1.333-2.915)$ & 0.001 \\
\hline
\end{tabular}

Logistic regression analysis was performed for metabolic syndrome at baseline, and Cox regression analysis was performed for metabolic syndrome development.

$\mathrm{OR}$, odds ratio; $\mathrm{Cl}$, confidence interval; HR, hazard ratio; LDL, low-density lipoprotein; CRP, C-reactive protein; MFG-E8, milk fat globule-EGF factor 8 protein.

(OR, 1.273; 95\% Cl, 1.183-1.369; $P<0.001)$, and MFG-E8 level $\geq 4,745.1 \mathrm{pg} / \mathrm{mL}$ (OR, 3.493; 95\% Cl, 2.298-5.311; $P<0.001$ ) were independent predictors for metabolic syndrome at baseline. In addition, higher $\mathrm{BMI}(\mathrm{HR}, 1.137 ; 95 \% \mathrm{Cl}, 1.067-1.212 ; P<0.001)$, MFG-E8 level $\geq 4,745.1 \mathrm{pg} / \mathrm{mL}$ (HR, 1.971; 95\% Cl, 1.333-2.915; $P=0.001)$ were significantly related to development of metabolic syndrome at follow-up.

\section{DISCUSSION}

The screening and prediction of metabolic syndrome are considered to be of great importance for public health; however, the useful single biomarker for metabolic syndrome has not been established yet. In this prospective cohort study, we investigated the diagnostic and predictive performance of serum MFG-E8 levels in metabolic syndrome. Subjects with serum MFG-E8 level $\geq 4,745.1$ $\mathrm{pg} / \mathrm{mL}$ exhibited a significantly higher risk for metabolic syndrome. The risk for further development of metabolic syndrome was also significantly higher in subjects with serum MFG-E8 level $\geq 4,745.1$ $\mathrm{pg} / \mathrm{mL}$. In addition, MFG-E8 level $\geq 4,745.1 \mathrm{pg} / \mathrm{mL}$ was an independent predictor for metabolic syndrome following adjustment for other important factors including smoking, alcohol status, and other biomarkers.
The best cut-off value of serum MFG-E8 level for the presence of metabolic syndrome was $4,745.1 \mathrm{pg} / \mathrm{mL}$ with a sensitivity of $41.9 \%$ and specificity of $82.5 \%$. When subjects were classified into two groups with this cut-off value, the prevalence of metabolic syndrome was significantly different between groups (63.9\% vs. $34.2 \%, P<0.001)$. In a previous study, adiponectin showed a $52.3 \%$ and $37.5 \%$ prevalence of metabolic syndrome in men and women, respectively, with its best cut-off value.' In another study, the AUROC of adiponectin for the diagnosis of metabolic syndrome was $0.682-0.644 .^{9}$ Compared to adiponectin, serum MFG-E8 could be a comparable or better surrogate marker for the diagnosis of metabolic syndrome. Recently, the leptin/adiponectin ratio showed an AUROC of 0.771-0.778 in the diagnosis of metabolic syndrome. ${ }^{8,9}$ Studies comparing these biomarkers in a single cohort are needed for further validation.

Serum MFG-E8 showed a significant correlation with all components of metabolic syndrome, including waist circumference, diastolic BP, serum total cholesterol, HDL-cholesterol, triglyceride, glucose levels, and pulse wave velocity (all $P<0.05$ ). In contrast, because adiponectin and leptin are adipokines closely linked to lipid metabolism, these markers had a lower correlation with glucose level, ${ }^{8}$ which is the major component of metabolic syndrome. MFG-E8 also had a significant association with pulse wave velocity in this study, which is in accordance with the results of previous 
studies, suggesting that MFG-E8 could be a promising diagnostic biomarker in vascular disease. ${ }^{19-22}$ It is suggested that MFG-E8 cause an age-associated increase in extracellular matrix adhesion molecules. Likewise, MFG-E8 could be useful not only in the diagnosis of metabolic syndrome but also in predicting its complication risk.

Among the patients without metabolic syndrome at baseline, the subgroup with MFG-E8 level $\geq 4,745.1 \mathrm{pg} / \mathrm{mL}$ had higher metabolic syndrome development rate than the other subgroup ( $55.4 \%$ vs. $34.5 \%, P=0.003)$. Considering the relatively short observational period of a median 17 months, this result suggests that subjects with higher MFG-E8 level should be closely monitored for metabolic syndrome development. Cho et al. ${ }^{23}$ investigated the predictive potential of adiponectin and visceral adipose tissue in the same cohort as the present study. During the same observational period, the highest incidence of metabolic syndrome was $46.15 \%$ in one subgroup, which is lower than that in the present study. In addition, considering the cost and convenience, serum MFG-E8 level seems to be more beneficial than the combination of adiponectin and visceral adipose tissue measurement.

We observed that the higher BMI and MFG-E8 level $\geq 4,745.1$ $\mathrm{pg} / \mathrm{mL}$ were independent predictors for both diagnosis and further development of metabolic syndrome (all $P<0.05$ ). This finding additionally supports the value of serum MFG-E8 as a diagnostic and predictive marker of metabolic syndrome. MFG-E8 is a peripheral membrane glycoprotein that is universally distributed and expressed abundantly in various organs. ${ }^{24}$ It has multiple functions including the clearance of apoptotic cells, ${ }^{25}$ suppression of proinflammatory cytokines, ${ }^{26}$ arterial remodeling and angiogenesis. $^{20,26}$ These various mechanisms play important role in progression of metabolic syndrome. ${ }^{27,28}$ To our knowledge, we firstly investigated the correlation of serum MFG-E8 level and metabolic syndrome. Previous studies have shown conflicting results regarding the correlation of MFG-E8 and pathological status in various diseases. ${ }^{29-31}$ In this study, a positive association was observed between serum MFG-E8 level and the presence of metabolic syndrome. This may be because the pathogenesis and organ damage of metabolic syndrome are complicated and involve various signaling pathways, in which MFG-E8 may play both proinflammatory and anti-inflammatory roles. Although the exact roles of MFGE8 in metabolic syndrome have not been clarified in this study, we believe that MFG-E8 mainly contributes to the proinflammatory role in these subjects with mild and early disease status, because subjects with a previous history of angina pectoris, myocardial in- farction, stroke, or any revascularization were excluded from this study.

This study has several limitations. First, the sample size was small for sufficient evaluation of the diagnostic performance of a biomarker. Clinical practice-based large, prospective studies are needed to assess its clinical utility on patient outcomes. Second, although this study was designed for a community-based cohort, enrollment was performed at a single center. Therefore, selection bias might be present, and this S-MAS cohort may not represent the general population in the community. Third, because of the short observation period and high rate of follow-up loss, the predictive performance of the biomarker could not be fully evaluated. Fourth, the serum MFG-E8 level was only measured at baseline, which limits its serial assessment with disease progression. Finally, the exact mechanism and role of MFG-E8 in disease development and progression has not been clarified and needs to be investigated in the future.

In conclusion, subjects with higher serum MFG-E8 level have a higher risk for metabolic syndrome and further development, even after adjusting for other important factors. Therefore, MFG-E8 could be an useful biomarker in screening for and serial monitoring of metabolic syndrome.

\section{Authors' contributions}

Conception and design: Y.S. Seo; Development of methodology: Y.S. Seo; Acquisition, analysis and interpretation of data: Y.S. Seo, H.J. Joo, and H.A. Lee; Writing, review, and/or revision of the manuscript: H.A. Lee, J. Lim, H.J. Joo, E.L.Yoon, Y.S. Lee, Y.K. Jung, J.H. Kim, H. An, H.J. Yim, Y.T. Jeen, J.E. Yeon, D. Lim, K.S. Byun, and Y.S. Seo; Administrative, technical, or material support: Y.S. Seo; Study supervision: Y.S. Seo.

\section{Acknowledgements}

This study was supported by "The Research Supporting Program of The Korean Association for the Study of the Liver and The Korean Liver Foundation", and "Grant from the Seoul Metropolitan Government".

The English in this manuscript has been checked by at least two professional editors. A specialist editor with suitable professional knowledge reviewed and corrected the English. An English language specialist subsequently checked the paper again. The first language of both editors is English. We would like to thank Editage (www.editage.co.kr) for English language editing. 


\section{Conflicts of Interest}

The authors have no conflicts to disclose.

\section{SUPPLEMENTARY MATERIAL}

Supplementary material is available at Clinical and Molecular Hepatology website (http://www.e-cmh.org).

\section{REFERENCES}

1. Mottillo S, Filion KB, Genest J, Joseph L, Pilote L, Poirier P, et al. The metabolic syndrome and cardiovascular risk a systematic review and meta-analysis. J Am Coll Cardiol 2010;56:1113-1132.

2. Ford ES. The metabolic syndrome and mortality from cardiovascular disease and all-causes: findings from the National Health and Nutrition Examination Survey II Mortality Study. Atherosclerosis 2004;173: 309-314.

3. Hydes TJ, Ravi S, Loomba R, Gray ME. Evidence-based clinical advice for nutrition and dietary weight loss strategies for the management of NAFLD and NASH. Clin Mol Hepatol 2020;26:383-400.

4. Grundy SM, Cleeman JI, Daniels SR, Donato KA, Eckel RH, Franklin $B A$, et al. Diagnosis and management of the metabolic syndrome: an American Heart Association/National Heart, Lung, and Blood Institute scientific statement. Curr Opin Cardiol 2006;21:1-6.

5. Alberti KG, Zimmet $P$, Shaw J. Metabolic syndrome--a new worldwide definition. A consensus statement from the International Diabetes Federation. Diabet Med 2006;23:469-480.

6. Alberti KG, Eckel RH, Grundy SM, Zimmet PZ, Cleeman JI, Donato $K A$, et al. Harmonizing the metabolic syndrome: a joint interim statement of the International Diabetes Federation Task Force on Epidemiology and Prevention; National Heart, Lung, and Blood Institute; American Heart Association; World Heart Federation; International Atherosclerosis Society; and International Association for the Study of Obesity. Circulation 2009;120:1640-1645.

7. Ryo M, Nakamura T, Kihara S, Kumada M, Shibazaki S, Takahashi M, et al. Adiponectin as a biomarker of the metabolic syndrome. Circ J 2004;68:975-981.

8. Yoon JH, Park JK, Oh SS, Lee KH, Kim SK, Cho IJ, et al. The ratio of serum leptin to adiponectin provides adjunctive information to the risk of metabolic syndrome beyond the homeostasis model assessment insulin resistance: the Korean Genomic Rural Cohort Study. Clin Chim Acta 2011;412:2199-2205.

9. Zhuo Q, Wang Z, Fu P, Piao J, Tian Y, Xu J, et al. Comparison of adiponectin, leptin and leptin to adiponectin ratio as diagnostic marker for metabolic syndrome in older adults of Chinese major cities. Dia- betes Res Clin Pract 2009;84:27-33.

10. Srikanthan K, Feyh A, Visweshwar H, Shapiro JI, Sodhi K. Systematic review of metabolic syndrome biomarkers: a panel for early detection, management, and risk stratification in the West Virginian population. Int J Med Sci 2016;13:25-38.

11. Esser N, Legrand-Poels S, Piette J, Scheen AJ, Paquot N. Inflammation as a link between obesity, metabolic syndrome and type 2 diabetes. Diabetes Res Clin Pract 2014;105:141-150.

12. Atabai K, Fernandez R, Huang X, Ueki I, Kline A, Li Y, et al. Mfge8 is critical for mammary gland remodeling during involution. Mol Biol Cell 2005;16:5528-5537.

13. Greenberg ME, Sun M, Zhang R, Febbraio M, Silverstein R, Hazen SL. Oxidized phosphatidylserine-CD36 interactions play an essential role in macrophage-dependent phagocytosis of apoptotic cells. J Exp Med 2006;203:2613-2625.

14. Khalifeh-Soltani A, McKleroy W, Sakuma S, Cheung YY, Tharp K, Qiu $Y$, et al. Mfge8 promotes obesity by mediating the uptake of dietary fats and serum fatty acids. Nat Med 2014;20:175-183.

15. Khalifeh-Soltani A, Gupta D, Ha A, Iqbal J, Hussain M, Podolsky MJ, et al. Mfge8 regulates enterocyte lipid storage by promoting enterocyte triglyceride hydrolase activity. JCI Insight 2016;1:e87418.

16. Raposo G, Stoorvogel W. Extracellular vesicles: exosomes, microvesicles, and friends. J Cell Biol 2013;200:373-383.

17. Théry C, Ostrowski M, Segura E. Membrane vesicles as conveyors of immune responses. Nat Rev Immunol 2009;9:581-593.

18. Colombo M, Raposo G, Théry C. Biogenesis, secretion, and intercellular interactions of exosomes and other extracellular vesicles. Annu Rev Cell Dev Biol 2014;30:255-289.

19. Ni YQ, Zhan JK, Liu YS. Roles and mechanisms of MFG-E8 in vascular aging-related diseases. Ageing Res Rev 2020;64:101176.

20. Fu Z, Wang M, Gucek M, Zhang J, Wu J, Jiang L, et al. Milk fat globule protein epidermal growth factor-8: a pivotal relay element within the angiotensin II and monocyte chemoattractant protein-1 signaling cascade mediating vascular smooth muscle cells invasion. Circ Res 2009;104:1337-1346.

21. Wang M, Jiang L, Monticone RE, Lakatta EG. Proinflammation: the key to arterial aging. Trends Endocrinol Metab 2014;25:72-79.

22. Zhao H, Zhang H, Qin X. Age-related differences in serum MFG-E8, TGF- $\beta 1$ and correlation to the severity of atherosclerosis determined by ultrasound. Mol Med Rep 2017;16:9741-9748.

23. Cho SA, Joo HJ, Cho JY, Lee SH, Park JH, Hong SJ, et al. Visceral fat area and serum adiponectin level predict the development of metabolic syndrome in a community-based asymptomatic population. PLoS One 2017;12:e0169289.

24. Oshima K, Aoki N, Kato T, Kitajima K, Matsuda T. Secretion of a peripheral membrane protein, MFG-E8, as a complex with membrane vesicles. Eur J Biochem 2002;269:1209-1218.

25. Andersen MH, Graversen H, Fedosov SN, Petersen TE, Rasmussen 
Han Ah Lee, et al. MFG-E8 is useful biomarker in metabolic syndrome

JT. Functional analyses of two cellular binding domains of bovine lactadherin. Biochemistry 2000;39:6200-6206.

26. Miksa M, Amin D, Wu R, Jacob A, Zhou M, Dong W, et al. Maturation-induced down-regulation of MFG-E8 impairs apoptotic cell clearance and enhances endotoxin response. Int J Mol Med 2008;22:743-748.

27. Kim YS, Kim SG. Endoplasmic reticulum stress and autophagy dysregulation in alcoholic and non-alcoholic liver diseases. Clin Mol Hepatol 2020;26:715-727.

28. Alegre NS, Garcia CC, Billordo LA, Ameigeiras B, Poncino D, Benavides J, et al. Limited expression of TLR9 on T cells and its functional consequences in patients with nonalcoholic fatty liver disease. Clin
Mol Hepatol 2020;26:216-226.

29. Hanayama R, Tanaka M, Miyasaka K, Aozasa K, Koike M, Uchiyama $Y$, et al. Autoimmune disease and impaired uptake of apoptotic cells in MFG-E8-deficient mice. Science 2004;304:1147-1150.

30. Sun G, Liu J, Xia G, Zhang L, Li Y, Zhou Z, et al. Reduced serum milk fat globule-epidermal growth factor 8 (MFG-E8) concentrations are associated with an increased risk of microvascular complications in patients with type 2 diabetes. Clin Chim Acta 2017;466:201-206.

31. Shimagaki T, Yoshio S, Kawai H, Sakamoto Y, Doi H, Matsuda M, et al. Serum milk fat globule-EGF factor 8 (MFG-E8) as a diagnostic and prognostic biomarker in patients with hepatocellular carcinoma. Sci Rep 2019;9:15788. 